International Journal of Engineering \& Technology, $7(4.10)(2018) 389-392$
International Journal of Engineering \& Technology
SPC
Website: www.sciencepubco.com/index.php/IJET
Research paper

\title{
Prime Labeling of Jahangir Graphs
}

\author{
Anantha Lakshmi.R ${ }^{1 *}$, Jayalakshmi.K ${ }^{2}$, Madhavi. $T^{3}$ \\ ${ }^{1}$ Research Scholar, JNTUA, Ananthapuramu, Andhra Pradesh, India- 515002 \\ ${ }^{2}$ JNTUA College of Engineering, Department of Mathematics, Ananthapuramu, Andhra Pradesh, India- 515002 \\ ${ }^{3}$ Anantha Lakshmi Institute of Technology, H\&S(Dept.),Ananthapuramu, Andhra Pradesh India-515721 \\ *Corresponding author E-mail: anantha.reddem@gmail.com
}

\begin{abstract}
The paper investigates prime labeling of Jahangir graph $J_{n, m}$ for $n \geq 2, m \geq 3$ provided that $n m$ is even. We discuss prime labeling of some graph operations viz. Fusion, Switching and Duplication to prove that the Fusion of two vertices $v_{l}$ and $v_{k}$ where $k$ is odd in a Jahangir graph $J_{n, m}$ results to prime graph provided that the product $n m$ is even and is relatively prime to $k$. The Fusion of two vertices $v_{n m+}$ ${ }_{l}$ and $v_{k}$ for any $k$ in $J_{n, m}$ is prime. The switching of $v_{k}$ in the cycle $C_{n m}$ of the Jahangir graph $J_{n, m}$ is a prime graph provided that $n m+l$ is a prime number and the switching of $v_{n m+l}$ in $J_{n, m}$ is also a prime graph .Duplicating of $v_{k}$, where $k$ is odd integer and $n m+2$ is relatively prime to $k, k+2$ in $J_{n, m}$ is a prime graph.
\end{abstract}

Keywords: Prime labeling; Jahangir graph; Fusion; Switching and duplication.

\section{Introduction}

The paper considers only finite simple undirected graph throughout. Prime labeling of a graph $G$ is a bijection $\quad$ f: $V(G) \rightarrow\{1$, $2, \ldots|V|\}$ such that $\operatorname{gcd}(f(u), f(v))=1$ for each edge $u v$. A graph is called prime graph if it admits a prime labeling. The graph $G$ has vertex set $V=V(G)$ and the edge set $E=E(G)$. The set of vertices adjacent to a vertex $u$ of $G$ is denoted as $N(u)$. For notation and terminology reference to Bondy and Murthy [1] has been made . Prime labeling is a concept that has been introduced by Roger Entringer . Since then many researchers have studied prime labeling for different types graphs. The cycle $C_{n}$ on $n$ vertices is a prime graph was proved by Dertsky [2]. Later Fu [3] considered path $P_{n}$ on $n$ vertices to show that such graphs are prime graphs. Roger surmised during the period 1980s that all trees possess prime labeling, and what he surmised could not be confirmed as a fact till now. Sundaram [8] is one of the exponents who studied the prime labeling for planner grid. Further investigations included the development of prime labelings by authors such as Ganesan and Balamurugan [4] who developed prime labellings for Theta graphs and Meena and Vaithilingam [6] for graphs related to Helm. In addition, Prime Labeling for several fan related graphs [7] have been proved by them. As far as cycle related graphs are concerned it was Vaidhya and K.K. Kanmani [9] who proved their prime labeling. Lee [5] has been attributed with establishing the fact that wheel $\mathrm{Wn}$ is a prime graph iff $n$ is even.

\section{Definition 1.1.}

Under specific conditions, when the vertices of the graphs have been demarcated with values then such phenomenon is termed as (vertex) graph labeling.

\section{Definition 1.2.}

Suppose $G=(V(\mathrm{G}), E(G))$ is a graph possessing $n$ vertices. A bijection $f: V(G) \rightarrow\{1,2, \ldots n\}$ is termed as Prime labeling, when $e=u v, \operatorname{hcf}(\mathrm{f}(\mathrm{u}), \mathrm{f}(\mathrm{v}))=1$ for each edge. When prime labeling occurs a graph is considered as prime graph.

\section{Definition 1.3.}

In a graph $\mathrm{G}$, the vertices which are an independent set are a set of interdependent vertices that are nonadjacent.

\section{Definition 1.4.}

Consider $u$ and $v$ are two separate vertices meant for a graph G. $\mathrm{G}_{1}$ is a novel graph that has been designed by fusing (identifying) the two vertices $u$ and $v$ by a sole vertex $x$ in $G_{1}$ in such a way that each edge that has been incident with either $u$ (or) $v$ in $G$ is at present incident with $x$ in $G_{1}$.

\section{Definition 1.5.}

A vertex switching $G_{v}$ of graphs $G$ has been procured by considering a vertex $v$ of $G$, deleting the total edges which are incident with $v$ and accumulating edges combining $v$ to each vertex that are not neighboring to $v$ in $G$.

\section{Definition 1.6.}

Duplication of a vertex $v$ of a graph $G$ produces a new graph G'by adding a vertex $\mathrm{v}^{\prime}$ with $\mathrm{N}(\mathrm{v})=\mathrm{N}\left(\mathrm{v}^{\prime}\right)$.

To define the other way a vertex $v^{\prime}$ is told to have been in duplication of $\mathrm{v}$ under condition that all the vertices that are beside $\mathrm{v}$ are at present neighbored to $\mathrm{v}^{\prime}$ In $\mathrm{G}^{\prime}$.

\section{Definition 1.7.}

Jahangir graph $\mathrm{J}_{\mathrm{n}, \mathrm{m}}$ for $\mathrm{n} \geq 2, \mathrm{~m} \geq 3$ is a graph with on $\mathrm{nm}+1$ vertices comprising a certain cycle $\mathrm{C}_{\mathrm{nm}}$ possessing single vertex that is additional and is beside $m$ vertices of $C_{n m}$ placed at a distance $\mathrm{n}$ between the $\mathrm{C}_{\mathrm{nm}}$. Jahangir graph $\mathrm{J}_{2,8}$ that is visible on his tomb which is located in his mausoleum. 


\section{Main Results of Prime Labeling on Jahangir Graph}

\section{Theorem 2. 1.}

If $n m$ is even then the Jahangir graph $J_{n, m}$ for $n \geq 2, m \geq 3$ is a prime graph.

\section{Proof:}

Let $J_{n, m}$ be a Jahangir graph. $V\left(J_{n, m}\right)=\left\{v_{1}, v_{2}, \ldots, v_{n m+1}\right\}$ and $E\left(J_{n, m}\right)$

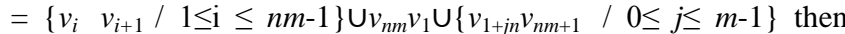
$\left|V\left(J_{n, m}\right)\right|=n m+1$ and $\left|E\left(J_{n, m}\right)\right|=(n+1) m$. Here the set $\left\{v_{i} v_{i+1} / 1 \leq\right.$ $i \leq n m-1\} \cup v_{n m} v_{1}$ represent as edges of the cycle and the set $\left.\mathrm{v}_{1+j \mathrm{n}} \mathrm{v}_{\mathrm{nm}+1} / 0 \leq j \leq m-1\right\}$ represent the set of edges adjacent to the vertex $v_{n m+1}$

The vertex labeling of $J_{n, m}$ is $f: V\left(J_{n, m}\right) \rightarrow\{1,2, \ldots, n m+1\}$ such that $f\left(v_{i}\right)=i+1$ for $1 \leq i \leq n m$ and $f\left(v_{n m+1}\right)=1$. It is to be noted that with $n m+1$ vertices and $n m+1$ labelings $f$ is bijection. As ' 1 ' is relatively prime to each natural number and any two successive natural numbers are relatively prime. Therefore, for each edge $e=$ $u v \in E\left(J_{n, m}\right)$ and $\operatorname{gcd}(f(u), f(v))=1$. Hence, $J_{n, m}$ proves to have undergone prime labeling. Therefore, $J_{n, m}$ is a prime graph.

\section{Illustration 2.2.}

The following graphs $1 \& 2$ indicates the Prime labeling of $\mathbf{J}_{2,3}$ and $\mathrm{J}_{3,4}$.

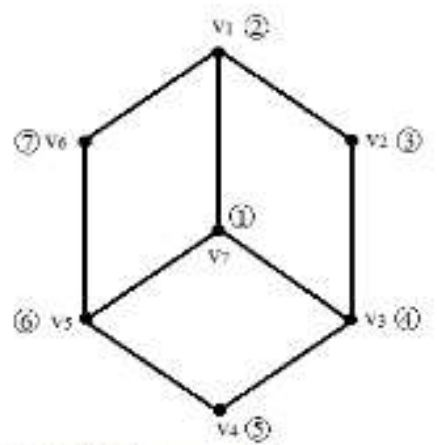

Figure 1. Jahtangir graph $\mathrm{J}_{23}$

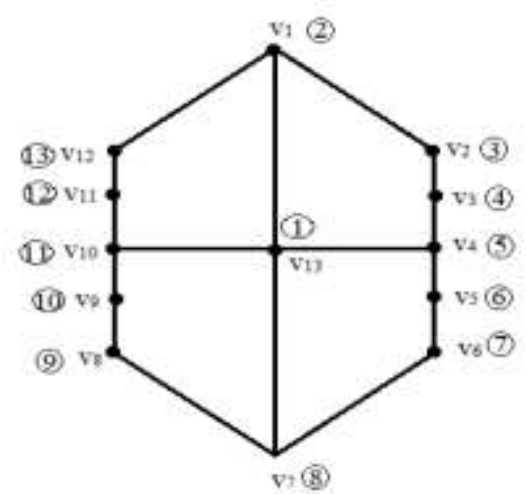

Figure 2. Jahangir graph $J_{3,4}$

\section{Programme 2.3.}

Pseudo code for the prime labeling of $\mathrm{J}_{\mathrm{n}, \mathrm{m}}$ is written in ' $\mathrm{C}$ ' programme

\#include<stdio.h>

\#include<conio.h>

int $\mathrm{f}$ (int);

intgcd(int,int);

intn,m;

void main ()

\{

inth,g,v[1000],e[1000],f1[1000],flag=1,i,j,flag $1=1 ;$;

clrscr();
printf("Enter n,m:");

scanf("\%d\%d",\&n,\&m);

for $(\mathrm{i}=1 ; \mathrm{i}<=\mathrm{n} * \mathrm{~m} ; \mathrm{i}++)$

\{

$\mathrm{f} 1[\mathrm{i}]=\mathrm{f}(\mathrm{i})$;

printf("\%d\n",f1[i]);

\}

for $(\mathrm{i}=1 ; \mathrm{i}<\mathrm{n} * \mathrm{~m} ; \mathrm{i}++)$

\{

$\mathrm{g}=\operatorname{gcd}(\mathrm{f} 1[\mathrm{i}], \mathrm{f} 1[\mathrm{i}+1])$;

printf("GCD=\%d",g);

if $(\mathrm{g} !=1)$

\{

flag $=0$;

break;

\}

\}

$\mathrm{h}=\operatorname{gcd}(\mathrm{f}(\mathrm{n} * \mathrm{~m}), \mathrm{f}(1))$;

printf("h=\%d",h);

for $(j=0 ; j<=m-1 ; j++)$

\{

$\mathrm{g}=\operatorname{gcd}(\mathrm{f}(1+\mathrm{j} * \mathrm{n}), \mathrm{f}(\mathrm{n} * \mathrm{~m}+1))$;

printf("GCD1=\%d",g);

if $(\mathrm{g} !=1)$

\{

flag $1=0$;

break;

\}

\}

printf("flag1=\%d",flag1);

if $($ flag $==1 \& \& \mathrm{~h}==1 \& \&$ flag $1==1)$

printf("In Prime Graph");

else

printf("In Not a Prime Graph");

getch();

\}

int $\mathrm{f}$ (int i)

\{

if $(\mathrm{i}==\mathrm{n} * \mathrm{~m}+1)$

return 1 ;

else

return $\mathrm{i}+1$;

\}

intgcd(inta,int b)

\{

int $\mathrm{r}$;

while $(b !=0)$

\{

$\mathrm{r}=\mathrm{a} \% \mathrm{~b}$

$\mathrm{a}=\mathrm{b}$;

$\mathrm{b}=\mathrm{r}$;

\}

return a;

\}

\section{Theorem 2.4.}

When $n m$ happens to be an odd number then $J_{n, m}$ will cease to be a prime graph.

Proof:

Note that the order of $J_{n, m}$ is $n m+1$. Hence, one has to use from 1 to $n m+1$ integers while labeling the vertices.In this way we have $n m+1$

I odd integers. For the moment, one can allocate odd numbers to at most $\frac{n m+\mathbf{1}}{\mathbf{2}}$ (as nm is odd) vertices from among the said $n m$ vertices in the cycle $C_{n m}$. Next one must assign a prime number to the center of the graph $J_{n, m}$ and each prime number is 
odd. Therefore, one has to assign at most places $\frac{n m+3}{2}$ odd numbers to the vertices. However, because we are having $\frac{n m+\mathbf{1}}{\mathbf{2}}$ odd numbers with us, it is not possible. Finally, $J_{n, m}$ is not considered to be a prime graph for odd $\mathrm{nm}$.

\section{Main Results on Fusion of Vertices in the Jahangir Graph $J_{n, M}$}

\section{Theorem 3.1.}

The Fusion of two vertices $v_{1}$ and $v_{k}$ in a Jahangir graph $J_{n, m}$ $\mathrm{n} \geq 2, \mathrm{~m} \geq 3$ such that $\mathrm{nm}$ is even and $\mathrm{nm}$ is relatively prime to $\mathrm{k}$ where $\mathrm{k}$ is an odd number is a prime graph.

\section{Proof:}

Suppose $\mathrm{G}$ is a graph resulting from fusion of two vertices $\mathrm{v}_{1}$ and $\mathrm{v}_{\mathrm{k}}$ where $\mathrm{k}$ is an odd number in the cycle of $\mathrm{J}_{\mathrm{n}, \mathrm{m}}$ then $|\mathrm{V}(\mathrm{G})|=\mathrm{nm}$ and $|\mathrm{E}(\mathrm{G})|=(\mathrm{n}+1) \mathrm{m}$. The set of edges are the edges which are incident on $\mathrm{v}_{1}$ and $\mathrm{v}_{\mathrm{k}}$ are incident with the new vertex ' $\mathrm{v}_{1}=\mathrm{v}_{\mathrm{k}}$ ', and the remaining are same.

We define the labeling $\mathrm{f}: \mathrm{V}(\mathrm{G}) \rightarrow\{1,2,3, \ldots \ldots \ldots \mathrm{nm}\}$ such that $\mathrm{f}\left(\mathrm{v}_{1}\right.$ $\left.=\mathrm{v}_{\mathrm{k}}\right)=\mathrm{k}$ and $\mathrm{f}\left(\mathrm{v}_{\mathrm{i}}\right)=\mathrm{i}$ for all $\mathrm{I}$ and $\mathrm{f}\left(\mathrm{v}_{\mathrm{nm}+1}\right)=1$. As ' $\mathrm{k}$ ' is an odd number so, the $\operatorname{gcd}(2, \mathrm{k})=1$ and $\mathrm{k}$ is relatively prime to $\mathrm{nm}$ and each pair of successive natural numbers are relatively prime. Therefore, $\operatorname{hcf}(\mathrm{f}(\mathrm{u}), \mathrm{f}(\mathrm{v}))=1$ for each edge $\quad \mathrm{e}=\mathrm{uv} \in \mathrm{E}(\mathrm{G})$. Hence, $\mathrm{G}$ complies prime labeling. Therefore $\mathrm{G}$ is a prime graph.

\section{Illustration 3.2.}

The following graphs represent the fusion of $\mathrm{v}_{1}$ and $\mathrm{v}_{3}$ in $\mathrm{J}_{2,5}$ and $\mathrm{v}_{1}$ and $\mathrm{v}_{5}$ in $\mathrm{J}_{3,4}$ respectively.
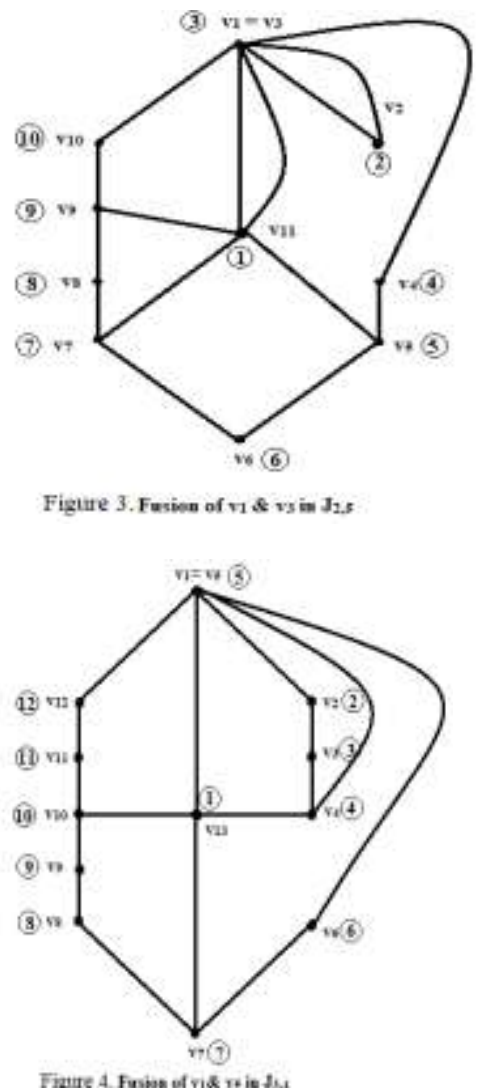

\section{Remark 3.3.}

The fusion of $v_{1}$ and $v_{3}$ in $J_{2,3}$ is prime even though 6 is not relatively prime to 3.The labeling of fusion of $v_{1}$ and $v_{3}$ in $J_{2,3}$ is shown in figure 5 .

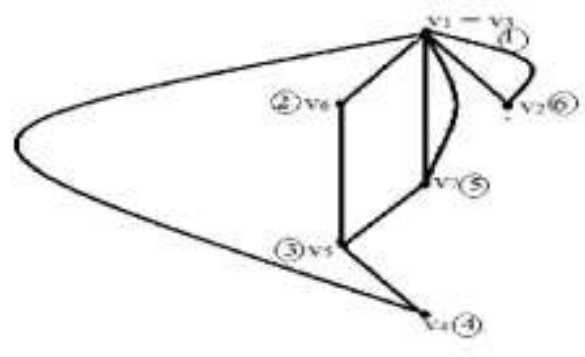

Figure 5. Fusion of $v_{1}$ and $v_{3}$ in $J_{23}$

Theorem 3.4.

The Fusion of two vertices $v_{n m+1}$ and $v_{k}$ for any $k$ in a Jahangir graph $J_{n, m}$ for $n \geq 2$ and $m \geq 3$ such that $n m$ is even is a prime graph.

Proof:

Suppose $\mathrm{G}$ is a graph resulting from fusion of two vertices $\mathrm{v}_{\mathrm{nm}+1}$ and $v_{k}$ in $J_{n, m}$ then $|V(G)|=n m$ and $|E(G)|=(n+1) m$. The set of edges in $\mathrm{G}$ are the set of all edges which are in the cycle $\mathrm{C}_{\mathrm{nm}}$ and the set of all edges which are adjacent to $\mathrm{v}_{\mathrm{nm}+1}$. Define the labeling $\mathrm{f}: \mathrm{V}(\mathrm{G}) \rightarrow\{1,2,3, \ldots, \mathrm{nm}\}$ such that $\mathrm{f}\left(\mathrm{v}_{\mathrm{nm}+1}=\mathrm{v}_{\mathrm{k}}\right)=1$ and $\mathrm{f}\left(\mathrm{v}_{\mathrm{k}-\mathrm{j}}\right)=$ $\mathrm{nm}+1-\mathrm{j}$ for $1 \leq \mathrm{j} \leq \mathrm{k}-1 ; \mathrm{f}\left(\mathrm{v}_{\mathrm{k}+\mathrm{i}}\right)=\mathrm{i}+1$ for $1 \leq \mathrm{i} \leq \mathrm{nm}-\mathrm{k}$. Note that $f(u), f(v)$ are co-prime numbers for each edge $e=u v \in E(G)$. Hence, $\mathrm{G}$ complies prime labeling. Therefore, $\mathrm{G}$ is a prime graph.

\section{Illustration 3.5.}

The fusion of $\mathrm{v}_{11}$ and $\mathrm{v}_{2}$, fusion of $\mathrm{v}_{11}$ and $\mathrm{v}_{4}$ in $\mathrm{J}_{2,5}$ shown in the figures 6 and 7

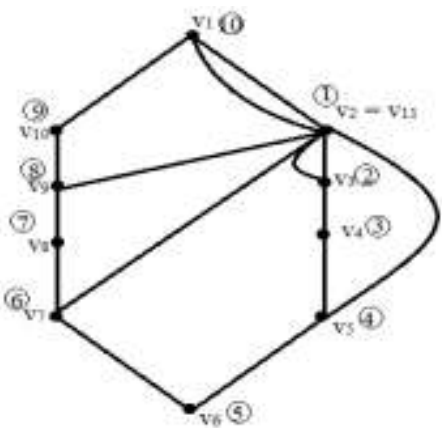

Figure 6. The fision of $v_{15}$ and $v_{2}$ in $J_{23}$

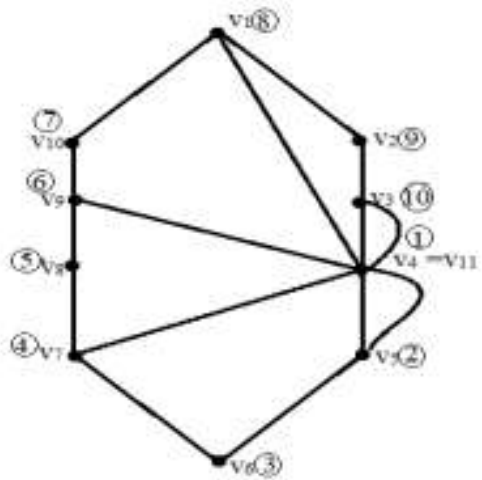

Figure 7 , The fusion of $\mathrm{v}_{11}$ and $\mathrm{v}_{4}$ in $\mathrm{J}_{2}, \mathrm{~s}$

\section{Main Results on Switching of Vertices in the Jahangir Graph $J_{n, M}$.}

\section{Theorem 4.1.}

The switching of $v_{n m+1}$ in the Jahangir graph $J_{n, m}$, for $n \geq 2, m \geq 3$ such that $n m$ is even is a prime graph. 


\section{Proof:}

Suppose $G$ is a graph resulting from switching $v_{n m+l}$ in $J_{n, m}$ then $|V(G)|=n m+1$ and $|E(G)|=(2 n-1) m$. The set of edges in $G$ are the set of edges in the cycle $C_{n m}$ and the set of edges which are not adjacent to $v_{n m+1}$. We define the labeling $f: V(G) \rightarrow\{1,2,3, \ldots, n m+1\}$ such that $f\left(v_{n m+1}\right)=1$ and $f\left(v_{i}\right)=i+1$ for $1 \leq i \leq n m$. Note that $f($ $u), f(v)$ are co- prime numbers for each edge $e=u v \in E(G)$. Hence, $G$ complies prime labeling. Therefore, $G$ is a prime graph

\section{Illustration 4.2.}

Switching of $\mathrm{v}_{13}$ in $\mathrm{J}_{3,4}$ is shown in the figure 8.

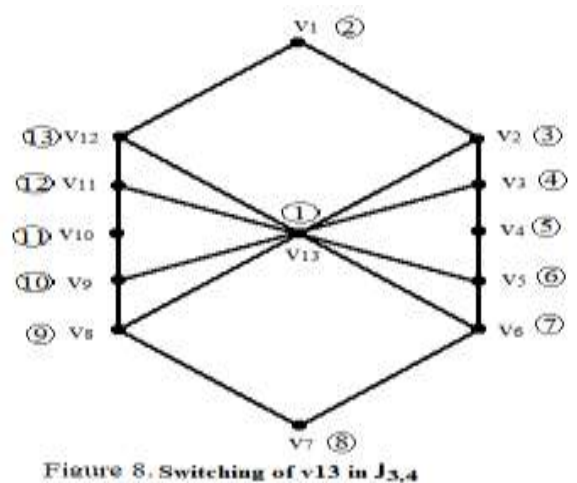

Theorem 4.3.

The switching of $v_{k} k \geq 1$, in the cycle $C_{n m}$ of the Jahangir graph $J_{n, m}$ for $n \geq 2, m \geq 3$ such that $n m+1$ is a prime number, is a prime graph.

\section{Proof:}

Suppose $G$ is a graph obtained by switching $v_{k}$ in $\mathrm{J}_{\mathrm{n}, \mathrm{m}}$ then $|V(G)|$ $=n m+1$. The set of edges in $G$ are the set of edges in the cycle $C_{n m}$ which are not incident on $v_{k}$ in $J_{n, m}$ are now incident on $v_{k}$ and the rest of edges will remain same in $J_{n, m}$. The required labeling $f: V(G) \rightarrow\{1,2,3, \ldots, n m+1\}$ such that $f\left(v_{k}\right)=n m+1, f\left(v_{n m+1}\right)=1$ and $f\left(v_{k+i}\right)=i+1$ for $1 \leq i \leq n m-k$ and $f\left(v_{k-i}\right)=n m+1-i$ for $1 \leq i \leq k-1$. Note that $f(u), f(v)$ are co-prime numbers for each edge $e=u v \in E(G)$.Hence, $G$ complies prime labeling. Therefore, $G$ is a prime graph

\section{Illustration 4.4.}

The switching of $v_{1}$ and switching of $v_{5}$ in $J_{3,4}$ are shown in the figures 9,10 .
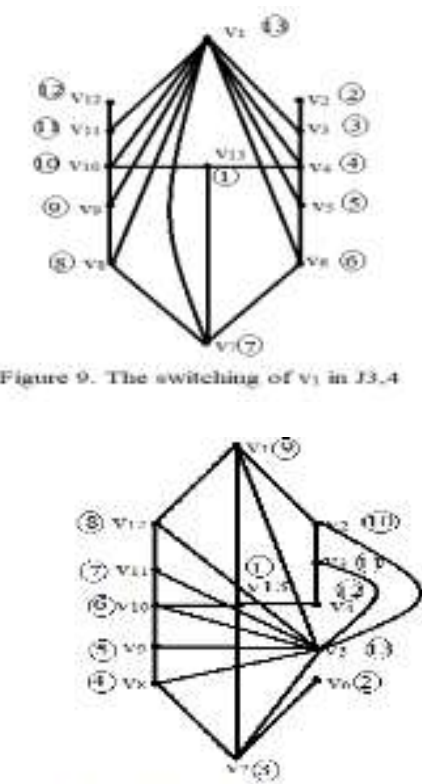

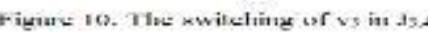

\section{Main Results on Duplication of a Vertex in the Jahangir Graph $J_{n, M}$.}

\section{Theorem 5.1.}

The Duplication of $v_{k}$ where $k$ is odd integer and $n m+2$ is relatively prime to $k, k+2$ in the Jahangir graph $J_{n, m}$, for $n \geq 2, m \geq 3$ such that $n m$ is even is a prime graph.

\section{Proof:}

Let $G$ be a graph obtained by duplicating $v_{k}$ by $v_{k}{ }^{\prime}$ in $J_{n, m}$. We define the labeling $f: V(G) \rightarrow\{1,2,3, \ldots, n m+2\}$ such that $f\left(v_{n m+1}\right)=1$ and $f\left(v_{i}\right)=i+1$ for $1 \leq i \leq n m, f\left(v_{k}{ }^{\prime}\right)=n m+2$. As mentioned in the theorem 2.1, $h c f(f(u), f(v))=1$ for each edge $e=$ $u v \in E(G)$. Hence, $G$ complies prime labeling. Therefore, $G$ is a prime graph.

\section{Illustration 5.2.}

Duplication of $v_{3}$ in $J_{3,4}$ is shown in the figure 11.

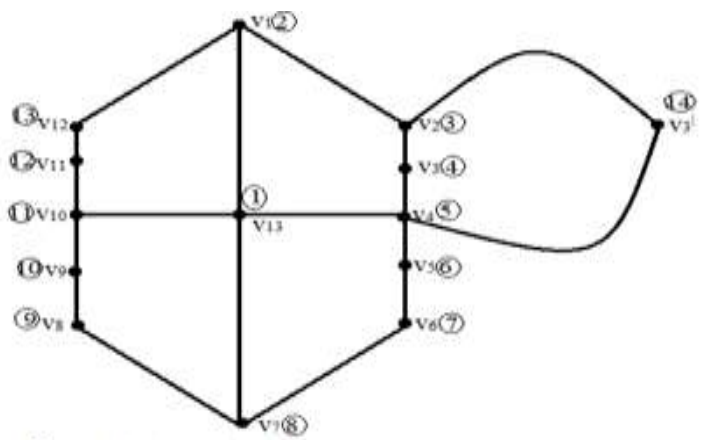

Figure 11. Duplication of $\mathrm{v}_{3}$ in $\mathrm{J}_{3} 4$

\section{Conclusion}

In this paper we checked the prime labeling of Jahangir graph $\mathrm{J}_{\mathrm{n}, \mathrm{m}}$ by using ' $C$ ' Program for different $n, m$ values which satisfy the condition $\mathrm{nm}$ is even. Similarly we can apply different languages for checking the labelings of different families of the graph.

\section{References:}

[1] J.A.Bondy and U.S.R.Murthy, Graph Theory and Applications (North Holland).New York (1976)

[2] T.O.Dretskyetal, "On Vertex Prime labeling of graphs in graph theory", Combinatories and applications vol.1 J.Alari (Wiley. N.Y. 1991)299-359

[3] H.C.Fu and K.C.Huany, "On Prime labeling Discrete Math”, 127 (1994) 181186

[4] V.Ganesan \& Dr.K.Balamurugan, "On prime labeling of Theta graph", International Journal of Current Research and Modern Education (IJCRME) ISSN (Online): 2455 - 5428 Volume I, Issue II, 2016

[5] S.M.Lee, L.Wui and J.Yen, "On the amalgamation of Prime graphs”. Bull. Malaysian Math.Soc. (Second Series) 11, (1988) 5967.

[6] S.Meena and K.Vaithilingam :Prime labeling for some helm related graphs, International Journal of Innovative Research in Science, Engineering and Technology Vol. 2, Issue 4, April 2013.

[7] S.Meena and K.Vaithilingam, "Prime labeling for some fan related graphs", International journal of Engineering Research \&technology (IJERT) ISSN :2278-0181 vol.1 Issue9,November2012.

[8] M.Sundaram Ponraj \& S.Somasundaram,( 2006) "On prime labeling conjecture are Combinatoria" 79 205-209

[9] S.K.Vaidya and K.K.Kanmani, "Prime labeling for some cycle related graphs", Journal of Mathematics Research vol.2. No.2.May 2010 (98-104) 